Mon. Not. R. Astron. Soc. 000, 16 6 (XXXX) Printed 20 January $2020 \quad$ (MN LATEX style file v2.2)

\title{
The radial acceleration relation in galaxy clusters
}

\author{
Man Ho Chan ${ }^{1 \star}$, Antonino Del Popolo ${ }^{2,3,4} \dagger$ \\ 1 Department of Science and Environmental Studies, The Education University of Hong Kong, Tai Po, Hong Kong \\ 2 Dipartimento di Fisica e Astronomia, University of Catania, Viale Andrea Doria 6, 95125 Catania, Italy \\ 3 INFN sezione di Catania, Via S. Sofia 64, I-95123 Catania, Italy \\ ${ }^{4}$ Institute of Astronomy RAS, Pyatnitskaya str., 48, Moscow, Russia
}

Accepted XXXX, Received XXXX

\begin{abstract}
Recently, the discovery of the radial acceleration relation (RAR) in galaxies has been regarded as an indirect support of alternative theories of gravity such as Modified Newtonian Dynamics (MOND) and modified gravity. This relation indicates a tight correlation between dynamical mass and baryonic mass in galaxies with different sizes and morphology. However, if the RAR relation is scale-independent and could be explained by alternative theories of gravity, this relation should be universal and true for galaxy clusters as well. In this article, by using the x-ray data of a sample of galaxy clusters, we investigate if there exists any tight correlation between dynamical mass and baryonic mass in galaxy clusters, assuming hot gas mass distribution almost representing baryonic distribution and that the galaxy clusters are virialized. We show that the resulting RAR of 52 non-cool-core galaxy clusters scatters in a large parameter space, possibly due to our simplifying assumptions and unclear matter content in galaxy clusters. This might indicate that the RAR is unlikely to be universal and scale-independent.
\end{abstract}

Key words: Dark matter

\section{INTRODUCTION}

The $\Lambda$ cold dark matter $(\Lambda \mathrm{CDM})$ model is well known to give very accurate predictions of the observations on cosmological scales, and intermediate scales (Komatsu et al. 2011; Spergel et al. 2003; Kowalski et al. 2008; Percival et al. 2010). To be precise, at cosmological scales the model 'suffers from the cosmological constant problem Weinberg 1989; Astashenok \& del Popolo 2012), and the cosmic coincidence problem, and at large scales several tensions are present, between the value of the Hubble parameter, $H_{0}$, and $\mathrm{SNe}$ Ia data, the 2013 Planck parameters (Planck Collaboration et al. 2014) and $\sigma_{8}$ obtained from cluster number counts and weak lensing. Also the Planck 2015 data are in tension with $\sigma_{8}$ growth rate (Macaulay et al. 2013), and with CFHTLenS weak lensing (Raveri 2015) data. Also the small scales (1 - $10 \mathrm{kpcs})$ are not devoid from issues (e.g., Moore 1994; Moore et al. 1999; Ostriker \& Steinhardt 2003; Bovlan-Kolchin et al. 2011, 2012; Oh et al. 2011; Del Popolo \& Hiotelis 2014; Del Popolo \& Le Delliou 2014, 2017; Zhou et al. 2017; Chang et al. 2018; Chang \& Zhou 2019), as the cusp/core problem (Moore 1994; Flores \& Primack 1994), the "miss- ing satellite problem" (Moore et al. 1999), the "Too-BigTo-Fail problem" (Garrison-Kimmel et al. 2013, 2014). Another issue of the $\Lambda \mathrm{CDM}$ model is the lack of detection of the dark matter component of the model (Tan et al. 2016; Akerib et al. 2017; Coolev 2017). Therefore, this long list of problems has ruled out a huge portion of the available parameter space of cold dark matter, especially for the most popular candidate - weakly interacting massive particles (WIMPs). This potentially challenges the standard cosmological model - the $\Lambda$ cold dark matter model (Merritt 2017).

Recently, the observed radial acceleration relation (RAR) in galaxies show a tight correlation between dark matter and baryons (McGaugh 2004; Lelli et al. 2016; McGaugh. Lelli \& Schombert 2016; Lelli et al. 2017). The observed scatter is remarkably small and these relations subsume and generalize some other relations, such as the Tully-Fisher relation (Lelli et al. 2017). Therefore, the RAR is claimed to be tantamount to a natural law (Lelli et al. 2017), which gives an additional challenge to the standard dark matter theory (Merritt 2017) 1. Here, we want to recall that another scaling law, namely the Mass-Temperature

\footnotetext{
1 Note that Navia (2018) found that the RAR is broken for galaxies with redshift $z>0.77$
}

^ chanmh@eduhk.hk
† adelpopolo@oact.inaf.it 
relation in galaxy clusters have been considered as a proof that we need modified gravity (Hammami \& Mota 2017), and later disproved (Del Popolo, Pace \& Mota 2019).

The proposed solutions to the $\Lambda \mathrm{CDM}$ model issues range from the proposal of modifying the nature of DM (Colín et al. 2000; Goodman 2000; Peebles 2000; Sommer-Larsen \& Dolgov 2001), or the power spectrum (e.g. Zentner \& Bullock 2003), to delegating the solution to the phenomena related to baryon physics (Navarro et al. 1996; Gelato \& Sommer-Larsen 1999; Read \& Gilmore 2005; Mashchenko et al. 2006; Governato et al. 2010 El-Zant et al. 2001, 2004; Romano-Díaz et al. 2008; Cole et al. 2011; Saburova \& Del Popolo 2014).

Another suggestion is that the missing mass in galaxies and galaxy clusters, and the issues of the $\Lambda$ CDM model can be explained by modifying the theory of gravity (Buchdahl 1970; Starobinskv 1980; Milgrom 1983b a; Ferraro 2012), without the help of dark matter. For example, the earliest version - Modified Newtonian Dynamics (MOND) suggests that the conventional Newton's second law should be modified when acceleration is smaller than a threshold value (Milgrom 1983b). Other versions such as Modified Gravity (MOG) (Moffat 2006), Galileon Gravity (GG) (Chan \& Hui 2018), Emergent Gravity (EG) (Verlinde 2017) and scale invariant theory (Maeder 2018) suggest that some extra terms appear in the gravitational law which can mimic the effect of dark matter. In particular, many theories of modified gravity (e.g. MOG, EG) give a specific connection between dynamical mass and baryonic mass. These theories may be able to account for the observations that the entire shapes of rotation curves of many galaxies trace their baryonic mass distributions.

Nevertheless, recent discovery of ultra-diffused galaxies lacking dark matter (NGC1052-DF2 and NGC1052DF4) (van Dokkum et al. 2018, 2019a) and dark matterdominated galaxy Dragonfly 44 (van Dokkum et al. 2019b) may potentially challenge the alternative theories of gravity. Most of the modified theories predict a larger dynamical mass while observations indicate that baryonic mass can nearly fully account for the dynamical mass van Dokkum et al. 2018, 2019a). This result reveals that the canonical stellar mass - halo mass relation (Moster, Naab \& White 2013) or the RAR may not be universal. However, some recent studies point out that the discovery of the dark matter-lacking galaxies and the Dragonfly 44 could be consistent with the theory of MOND Kroupa et al. 2018a; Famaey, McGaugh \& Milgrom 2018; Haghi et al. 2019a b). Besides, the uncertainties of the distance of these dark-matter-lacking galaxies also make this issue more controversial (Truiillo et al. 2019; Monelli \& Truiillo 2019; Haslbauer et al. 2019). If these galaxies lie at about $10 \mathrm{Mpc}$, then their stellar masses go down significantly and the ratio between dynamical and stellar mass increases such that these galaxies would become normal dwarf galaxies.

Furthermore, some recent studies show that the existence of a fundamental acceleration $a_{0}$ claimed in RAR is rejected at more than $10 \sigma$ (Rodrigues et al. 2018a). It means that any alternative to dark matter based on the existence of a fundamental acceleration scale is ruled out. However, recent analyses continue to support the Milgromian accel- eration scale (McGaugh et al. 2018; Kroupa et al. 2018b) and the debate is still on-going (Rodrigues et al. 2018b).

In this article, we examine the possible RAR for galaxy cluster scale. If there exists a universal fundamental acceleration scale claimed by some modified gravity theories, the RAR should be universal for both galaxies and galaxy clusters. We use the x-ray data of 52 non-cool-core galaxy clusters and show that the RAR is unlikely to be universal. The resulting scatter is too large to be treated as a standard or law-like relation.

\section{THE RADIAL ACCELERATION RELATION}

Recent fits of the Spitzer Photometry \& Accurate Rotation Curves (SPARC) and some other dwarf spheroidal and earlytype galaxies data reveal a strong correlation between the radial dynamical acceleration $\left(a_{\mathrm{dyn}}=v^{2} / r\right)$ and the radial acceleration predicted by the observed distribution of baryons $\left(a_{\mathrm{bar}}=\partial \Phi_{b} / \partial r\right.$, where $\Phi_{b}$ is the gravitational potential of the baryonic component) (McGaugh, Lelli \& Schombert 2016; Lelli et al. 2017). It is called the radial acceleration relation (RAR), which is a particular form of the massdiscrepancy-acceleration (MDA) relation (McGaugh 2004). Another similar relation proposed in Lelli et al. (2016), the central-surface-densities relation, is also closely related to the RAR. The RAR can be well described by the following empirical function (McGaugh. Lelli \& Schombert 2016)

$$
a_{\mathrm{dyn}}=\frac{a_{\mathrm{bar}}}{1-e^{-\sqrt{a_{\mathrm{bar}} / a_{0}}}}
$$

where $a_{0}=1.20 \pm 0.02($ random $) \pm 0.24$ (systematic) $\times 10^{-10}$ $\mathrm{m} \mathrm{s}^{-2}$ is a constant. Later, Milgrom (2016) shows that the theory of MOND can give a natural explanation to the observed central-surface-densities relation. Also, McGaugh, Lelli \& Schombert (2016) argue that the RAR observed is consistent with MOND's prediction. Generally speaking, the existence of strong correlation between dark matter and baryons favors the alternative theories of gravity (Milgrom 2016).

At the same time, Desmond (2017); Chan (2017); Ludlow et al. (2017) show that the standard dark matter theory can also give the RAR. However, the scatter in the RAR cannot be completely reproduced by a cold dark matter (CDM) model (Desmond 2017). Also, some parameters or feedbacks have to be adjusted to match the observations (Ludlow et al. 2017). Besides, a recent study using the data of six deep imaging and spectroscopic surveys shows that the intrinsic scatter of the stellar RAR could be as small as 0.11 $\operatorname{dex}$ (Stone \& Courteau 2019). Another uncertainty is that the characteristic acceleration in the RAR could decrease by $40 \%$ with a scatter around 0.12 dex if we include the effect of cold dark baryons (Ghari, Haghi \& Hasani Zonoozi 2019). Therefore, this issue is very complicated and it is still controversial to claim the RAR as tantamount to a natural law (Lelli et al. 2017). In the following, we explore the RAR in galaxy clusters to see whether it is a universal relation. If it appears with the similar form on a larger scale (galaxy cluster scale), this may give an extra indirect evidence for the claim and favour the alternative theories of gravity. Note that although RAR is consistent with MOND's prediction and it is known that MOND works poorly in galaxy clusters 
(Ferreras et al. 2012), it does not mean that galaxy clusters do not have a similar RAR. Here, we are going to examine whether there exists a universal acceleration scale (exhibited in the form of RAR) in both galaxies and galaxy clusters as predicted by some modified gravity theories.

Using the data of galaxy clusters in Chen et al. (2007), we can obtain the dynamical acceleration $a_{\mathrm{dyn}}$ and the "baryonic acceleration' $a_{\text {bar. }}$. By assuming hydrostatic equilibrium, the pressure gradient of hot gas in a galaxy cluster is balanced by the gravitational force via the Newton's second law:

$$
\frac{d P}{d r}=-\rho_{g}(r) a_{\mathrm{dyn}},
$$

where $P=\rho_{g}(r) k T / m_{g}, \rho_{g}(r)$ is the hot gas mass density and $m_{g}$ is the average mass of a hot gas particle. Here, the term $a_{\text {dyn }}$ is not the real acceleration. It represents the strength of the gravity. Although we are not considering the dynamical acceleration inside a galaxy cluster, the physical meaning of $a_{\text {dyn }}$ truly represents the acceleration of a particle if it is placed at a particular $r$ due to gravitational attraction. But in actual situation, the gravitational attraction of hot gas particles are balanced by the gas pressure. If the gas pressure is absent, $a_{\mathrm{dyn}}$ is the actual acceleration of the gas particles. In other words, the term $a_{\text {dyn }}$ represents the 'potential acceleration' due to the gravitational attraction of the dynamical mass in a galaxy cluster.

For theories of modified gravity such as MOG and EG, Eq. (2) holds as these theories agree with the Newton's second law. However, the expression of $a_{\mathrm{dyn}}$ for modified gravity is different from the conventional Newtonian gravitation $a_{\text {dyn }}=G M / r^{2}$, where $M$ is the total enclosed mass. The only assumption here is using the hydrostatic equilibrium in the calculations. Recent studies point out that using x-ray hydrostatic mass measurements would give only $15-20 \%$ systematic uncertainty (Biffi et al. 2016), which is acceptable compared with the measurement errors of the parameters used.

Observations indicate that the temperature profiles of hot gas in many galaxy clusters are close to constant, except for their inner regions of cool-core clusters (Reiprich \& Böhringer 2002; Chen et al. 2007). Therefore, using a constant temperature profile for each non-coolcore galaxy cluster is a very good assumption. The overall percentage error of mass estimation is less than $15 \%$ (Vikhlinin et al. 2006). The density profile of hot gas can be described by a $\beta$-model (Cavaliere \& Fusco-Femiano 1976, 1978; Chen et al. 2007):

$$
\rho_{g}=\rho_{0}\left(1+\frac{r^{2}}{r_{c}^{2}}\right)^{-3 \beta / 2},
$$

where $\rho_{0}, r_{c}$ and $\beta$ are fitted parameters. By putting Eq. (3) into Eq. (2), we get

$$
a_{\mathrm{dyn}}=\frac{3 \beta k T r}{m_{g}\left(r^{2}+r_{c}^{2}\right)} .
$$

On the other hand, although the hot gas in galaxy clusters is not really accelerating inward, we define the baryonic acceleration term by

$$
a_{\mathrm{bar}}=\frac{G M_{\mathrm{bar}}}{r^{2}}=\frac{4 \pi G}{r^{2}} \int_{0}^{r} \rho_{g} r^{\prime 2} d r^{\prime} .
$$

The term $a_{\mathrm{bar}}$ as a function of $r$ for each galaxy cluster
Table 1. Scatter budget for the RAR in galaxy clusters.

\begin{tabular}{ll}
\hline Source & Residual \\
\hline Errors in $T$ & $0.07 \mathrm{dex}$ \\
Errors in $r_{c}$ & $0.06 \mathrm{dex}$ \\
Errors in $\beta$ & $0.08 \mathrm{dex}$ \\
Errors in $\rho_{0}$ & $0.06 \mathrm{dex}$ \\
\hline Total & $0.13 \mathrm{dex}$ \\
\hline
\end{tabular}

can be integrated numerically. It represents the gravitational strength due to baryonic mass.

To examine the RAR in galaxy clusters, we choose 52 non-cool-core clusters with $r_{c} \geqslant 100 \mathrm{kpc}$ in Chen et al. (2007) for analysis. We have ruled out small galaxy clusters because many of them are dominated by the bright cluster galaxies (BCGs) which may induce large systematic uncertainties in hydrostatic mass calculations. By using the parameters obtained in Chen et al. (2007), we can get $a_{\mathrm{dyn}}$ and $a_{\text {bar }}$ as a function of $r$. 2 We determine four different positions for each galaxy cluster (at $r=r_{c}, r=2 r_{c}, r=3 r_{c}$ and $r=r_{500}$, where $r_{500}$ is the position where average mass density equals 500 times of the cosmological critical density) to calculate the RAR.

We plot the graph $a_{\text {dyn }}$ against $a_{\text {bar }}$ to illustrate the RAR in galaxy clusters (Fig. 1). We can see that the resulting RAR for different galaxy clusters scatters in a large parameter space. By comparing with the RAR in galaxies, they have only a very little overlap in the low acceleration regime. Furthermore, the scatter is very large in the small range of $a_{\text {bar }}$. The resulting scatter is $0.18 \mathrm{dex}$, which is larger than the scatter of the RAR in galaxies (0.13 dex) (McGaugh, Lelli \& Schombert 2016). By considering the scatter budget of the involved parameters, the total expected scatter is 0.13 dex (see Table 1). Hence, the intrinsic scatter of the RAR is significant. This suggests that the RAR is not a universal relation and no universal acceleration scale exists. We also fit the data by using Eq. (1) (see Fig. 2). The best-fit value of the acceleration constant is $a_{0}=9.5 \times 10^{-10}$ $\mathrm{m} \mathrm{s}^{-2}$ for the overall empirical RAR, which is much larger than the expected acceleration constant $a_{0} \sim 1 \times 10^{-10} \mathrm{~m}$ $\mathrm{s}^{-2}$. This is consistent with MOND prediction in galaxy cluster scale (Sanders \& McGaugh 2002; Famaev \& McGaugh 2012). This may also suggest that there is no universal acceleration scale, or, some additional hot dark matter is needed in galaxy clusters. By plotting the RAR for different subsamples (at $r_{c}, 2 r_{c}$ and $3 r_{c}$ ) in Fig. 2, the corresponding $a_{0}$ are $1.9 \times 10^{-9} \mathrm{~m} \mathrm{~s}^{-2}$ (at $r_{c}$ ), $1.2 \times 10^{-9} \mathrm{~m} \mathrm{~s}^{-2}$ (at $2 r_{c}$ ) and $3.9 \times 10^{-10} \mathrm{~m} \mathrm{~s}^{-2}$ (at $3 r_{c}$ ). We can see the trend of larger discrepancies from the galactic RAR as one gets closer to the centres of galaxy clusters. This may indicate the significant effects of astrophysical processes near the centres of galaxy clusters.

2 The parameters used are re-scaled because the Hubble parameter assumed in Chen et al. (2007) is $h=0.5$ while we have used $h=0.68$ in our analysis. 


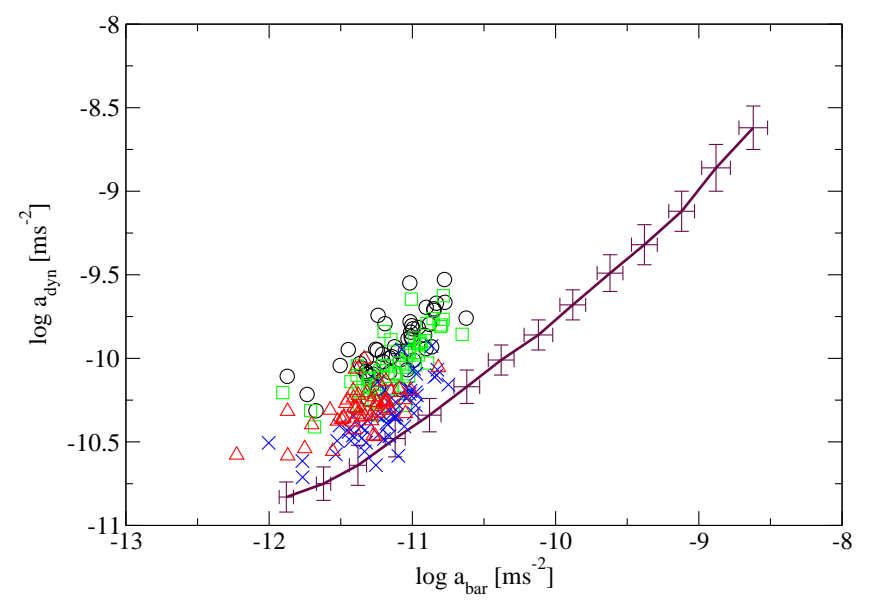

Figure 1. The black circles, green squares, blue crosses and red triangles indicate the RAR for 52 non-cool-core galaxy clusters at $r=r_{c}, r=2 r_{c}, r=3 r_{c}$ and $r=r_{500}$ respectively. The data points with error bars are the RAR of spiral galaxies (McGaugh, Lelli \& Schombert 2016).

\section{DISCUSSION}

Previous study using 13 galaxy clusters show that the MDA relation or RAR in galaxy clusters is less clear than that for galaxies (Edmonds et al. 2018). In our study, we investigate a larger sample and show that the resulting RAR for 52 non-cool-core galaxy clusters scatters in a large parameter space. The overall scatter is large and it does not agree with the RAR for spiral galaxies. In other words, the RAR is not a universal relation and it is not scale-independent. Since the RAR gives a specific connection between dynamical mass and baryonic mass, if the RAR is not universal, the connection between dynamical mass and baryonic mass may not be universal either.

In this study, we assume that the galaxy clusters are virialised and the corresponding hot gas is in hydrostatic equilibrium. Generally speaking, this is a good assumption and the systematic uncertainty involved is about $15-20 \%$ (Biffi et al. 2016), which is acceptable compared with the scatters. Furthermore, the hot gas mass does not completely represent all baryonic mass in a galaxy cluster. Strictly speaking, some cool gas clouds may exist which could not be detected by x-ray observations. This may make the assumptions about the baryonic distribution less secure. Therefore, if the above simplifying assumptions do not hold, the results in this study may need to revise. On the other hand, the uncertainties of the hot gas temperature profiles and some astrophysical processes like supernovae may also affect the scatters of the RAR in galaxy clusters. More in-depth investigations using simulations or astronomical observations might be required to tackle these issues.

Since there is no universal acceleration scale, the tight RAR in spiral galaxies cannot be treated as an evidence for the alternative theories of gravity. The tight correlation between dynamical mass and baryonic mass in galaxies may be

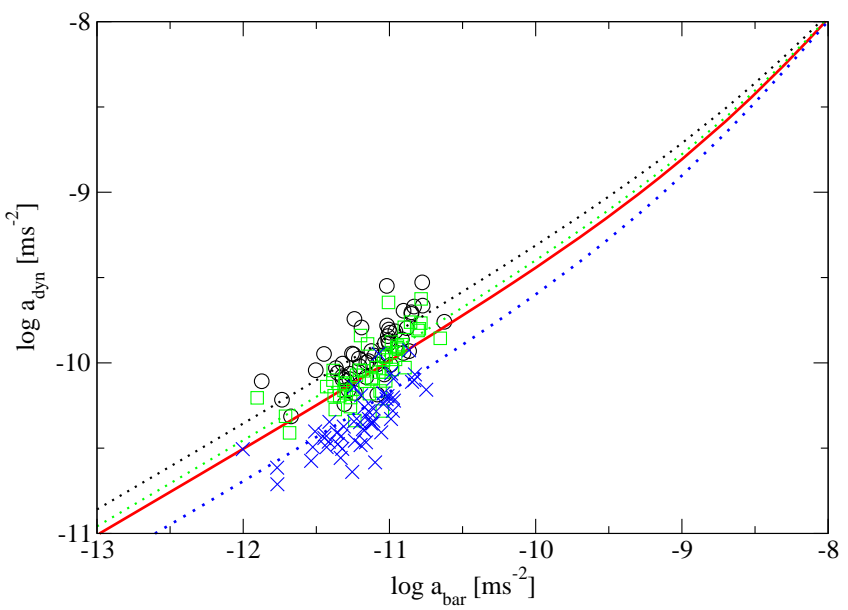

Figure 2. The red solid line indicates the overall empirical RAR following Eq. (1). The black circles, green squares and blue crosses indicate the RAR for 52 non-cool-core galaxy clusters at $r=r_{c}$, $r=2 r_{c}$ and $r=3 r_{c}$ respectively. The corresponding coloured dotted lines represent the best-fit RAR at $r_{c}, 2 r_{c}$ and $3 r_{c}$ respectively. The best-fit value of $a_{0}$ for the overall empirical RAR is $9.5 \times 10^{-10} \mathrm{~m} \mathrm{~s}^{-2}$.

due to some other reasons, such as dark matter-baryon interaction (Famaev, Khoury \& Penco 2018) or baryonic feedback processes (Pontzen \& Governato 2014). Recent discovery of the dark-matter-lacking galaxy indicates that the correlation between dynamical mass and baryonic mass in galaxies is not necessarily true (van Dokkum et al. 2018). Our result basically supports this new finding, though it is still controversial to claim that the galaxy is completely lacking dark matter (Martin et al. 2018).

Although some studies show that RAR might be closely related to MOND (McGaugh, Lelli \& Schombert 2016), our analysis does not have any implication on MOND. It is because MOND does not agree with the conventional Newton's second law when the acceleration is lower than $\sim 10^{-10} \mathrm{~m}$ $\mathrm{s}^{-2}$. Therefore, Eq. (2) has to be modified in the MOND framework. Nevertheless, it is well known that MOND works poorly in galaxy clusters. Many studies point out that MOND cannot account for the dynamical mass (missing mass) in galaxy clusters (Sanders \& McGaugh 2002). Also, recent radio tracking data of the Cassini spacecraft shows no deviation from General Relativity and it excludes a large part of the relativistic MOND theories (Hees et al. 2014). Some studies show that the apparent behavior of MOND can be explained by dark matter theory Dunkel 2004; Chan 2013), which means MOND may be just a phenomenological description rather than a universal theory. However, the problem of MOND in galaxy clusters or in cosmological scale might be solved by considering relativistic extensions of MOND (Tensor-Vector-Scalar TeVeS theory). Recent analysis shows that some relativistic extensions of MOND can satisfy the stringent constraints of the speed of gravitational waves (Skordis \& Zlosnik 2019). Some other studies point 
out that the problem of MOND in galaxy clusters could be alleviated if there exists some massive sterile neutrinos in our universe (Angus et al. 2010). Adding sterile neutrinos to MOND might be able to account for the RAR in galaxy clusters because the 'baryonic mass' could be increased by a certain factor such that the empirical RAR would be fitted to the galaxy cluster data with the canonical value of $a_{0}$. The possibility of the existence of massive sterile neutrinos is hinted by some ground-based experiments (Aguilar-Arevalo et al. 2018) and now becoming a hot issue in particle physics. It is also possible to have some connections between MOND and long-range quantum gravity (Cadoni \& Tuveri 2019). Other versions of MOND like "extended MOND" (EMOND) in which the acceleration scale depends on the depth of gravitational potential can explain the dynamics of galaxy clusters (Zhao \& Famaey 2012). Therefore, MOND is still a popular alternative theory of gravity currently. Further astronomical observations or cosmological studies are required to settle this controversial issue.

\section{ACKNOWLEDGEMENTS}

We are grateful to the referee for helpful comments on the manuscript. This work was supported by a grant from the Research Grants Council of the Hong Kong Special Administrative Region, China (Project No. EdUHK 28300518) and the grants from the Education University of Hong Kong (RG2/2019-2020R and RG7/2019-2020R).

\section{REFERENCES}

Aguilar-Arevalo A. A. et al., 2018, Phys. Rev. Lett. 121, 221801.

Akerib D. S. et al. [LUX Collaboration], 2017, Phys. Rev. Lett. 118, 021303.

Angus G. W., Famaey B. \& Diaferio A., 2010, Mon. Not. R. Astron. Soc. 402, 395.

Astashenok A. V., del Popolo A., 2012, Classical and Quantum Gravity, 29, 085014

Biffi V. et al., 2016, Astrophys. J. 827, 112.

Boylan-Kolchin M., Bullock J. S., Kaplinghat M., 2011, MNRAS, 415, L40

Boylan-Kolchin M., Bullock J. S., Kaplinghat M., 2012, MNRAS, 422, 1203

Buchdahl H. A., 1970, MNRAS, 150, 1

Cadoni M. \& Tuveri M., 2019, Phys. Rev. D 100, 024029.

Cavaliere A., Fusco-Femiano R., 1976, A\&A, 49, 137

Cavaliere A., Fusco-Femiano R., 1978, A\&A, 70, 677

Chan M. H., 2013, Phys. Rev. D 88, 103501.

Chan M. H., 2017, Int. J. Mod. Phys. D 26, 1750118.

Chan M. H. \& Hui H. K., 2018, Astrophys. J., 856, 177.

Chang Z., Lin H.-N., Zhao Z.-C., Zhou Y., 2018, Chin. Phys., C42, 115103

Chang Z., Zhou Y., 2019, MNRAS, 486, 1658

Chen Y., Reiprich T. H., Böhringer H., Ikebe Y. \& Zhang Y.-Y., 2007, Astron. Astrophys. 466, 805.

Cole D. R., Dehnen W., Wilkinson M. I., 2011, MNRAS, 416, 1118
Colín P., Avila-Reese V., Valenzuela O., 2000, ApJ, 542, 622

Cooley J. A., 2017, Physics 10, 3.

Del Popolo A., Hiotelis N., 2014, J. Cosmology Astropart. Phys., 1, 47

Del Popolo A., Le Delliou M., 2014, J. Cosmology Astropart. Phys., 2014, 051

Del Popolo A., Le Delliou M., 2017, Galaxies, 5, 17

Del Popolo A., Pace F. \& Mota D. F., 2019, Phys. Rev. D $100,024013$.

Desmond H., 2017, Mon. Not. R. Astron. Soc. 464, 4160.

Dunkel J., 2004, Astrophys. J. 604, L37.

Edmonds D., Farrah D., Minic D., Ng Y. J. \& Takeuchi T., 2018, arXiv:1801.00160.

El-Zant A., Shlosman I., Hoffman Y., 2001, ApJ, 560, 636

El-Zant A. A., Hoffman Y., Primack J., Combes F., Shlosman I., 2004, ApJ, 607, L75

Famaey B., Khoury J. \& Penco R., 2018, J. Cosmol. Astropart. Phys. 03, 038.

Famaey B., McGaugh S. \& Milgrom M., 2018, Mon. Not. R. Astron. Soc. 480, 473.

Famaey B. \& McGaugh S., 2012, Living Reviews in Relativity $15,10$.

Ferreras I., Mavromatos N. E., Sakellariadou M. \& Yusaf M. F., 2012, Phy. Rev. D 86, 083507.

Ferraro R., 2012, 1471, 103

Flores R. A., Primack J. R., 1994, ApJ, 427, L1

Garrison-Kimmel S., Boylan-Kolchin M., Bullock J. S., Kirby E. N., 2014, MNRAS, 444, 222

Garrison-Kimmel S., Rocha M., Boylan-Kolchin M., Bullock J. S., Lally J., 2013, MNRAS, 433, 3539

Gelato S., Sommer-Larsen J., 1999, MNRAS, 303, 321

Ghari A., Haghi H. \& Hasani Zonoozi A., 2019, Mon. Not. R. Astron. Soc. 487, 2148.

Goodman J., 2000, New A, 5, 103

Governato F., Brook C., Mayer L., Brooks A., Rhee G., Wadsley J., Jonsson P., Willman B., Stinson G., Quinn T., Madau P., 2010, Nature, 463, 203

Haghi H. et al., 2019a, Mon. Not. R. Astron. Soc. 487, 2441. Haghi H., Amiri V., Hasani Zonoozi A., Banik I., Kroupa P. \& Haslbauer M., 2019b, Astrophys. J. 884, L25.

Hammami A., Mota D. F., 2017, A\&A, 598, A132

Haslbauer M., Banik I., Kroupa P. \& Grishunin K., 2019, Mon. Not. R. Astron. Soc. 489, 2634.

Hees A., Folkner W. M., Jacobson R. A. \& Park R. S., 2014, Phys. Rev. D 89, 102002.

Komatsu E., Smith K. M., Dunkley J., Bennett C. L., Gold B., Hinshaw G., Jarosik N., Larson D., Nolta M. R., Page L., 2011, ApJS, 192, 18

Kowalski M., Rubin D., Aldering G., Agostinho R. J., Amadon A., Amanullah R., Balland C., Barbary K., Blanc G., Challis P. J., Conley A., Connolly N. V. e. a., 2008, ApJ, 686, 749

Kroupa P. et al., 2018a, Nature 561, E4.

Kroupa P. et al., 2018b, Nat. Astron. 2, 925.

Lelli F., Fraternali F., Sancisi R., 2010, A\&A, 516, A11

Lelli F., McGaugh S. S., Schombert J. M. \& Pawlowski M. S., 2016, Astrophys. J. 827, L19.

Lelli F., McGaugh S. S., Schombert J. M. \& Pawlowski M. S., 2017, Astrophys. J. 836, 152.

Ludlow A. D. et al., 2017, Phys. Rev. Lett. 118, 161103. Macaulay E., Wehus I. K., Eriksen H. K., 2013, Physical 
Review Letters, 111, 161301

Maeder A., arXiv:1804.04484.

Martin N. F., Collins M. L. M., Longeard N. \& Tollerud E., arXiv:1804.04136

Mashchenko S., Couchman H. M. P., Wadsley J., 2006, Nature, 442, 539

McGaugh S. S., 2004, Astrophys. J. 609, 652.

McGaugh S. S., 2011, Physical Review Letters, 106, 121303

McGaugh S. S., Lelli F. \& Schombert J. M., 2016, Phys.

Rev. Lett. 117, 201101.

McGaugh S. S., Li P., Lelli F. \& Schombert J. M., 2018, Nat. Astron. 2, 924.

Merritt D., 2017, Studies in History and Philosophy of Modern Physics 57, 41.

Milgrom M., 1983a, ApJ, 270, 371

Milgrom M., 1983b, ApJ, 270, 365

Milgrom M., 2016, Phys. Rev. Lett. 117, 141101.

Moffat J. W., 2006, J. Cosmol. Astro. Phys. 03, 004.

Monelli M. \& Trujillo I., arXiv:1907.03761.

Moore B., 1994, Nature, 370, 629

Moore B., Quinn T., Governato F., Stadel J., Lake G., 1999, MNRAS, 310, 1147

Moster B. P., Naab T. \& White S. D. M., 2013, Mon. Not.

R. Astron. Soc., 428, 3121.

Navarro J. F., Eke V. R., Frenk C. S., 1996, MNRAS, 283, L72

Navia C. E., 2018, arXiv e-prints, p. arXiv:1804.05914

Oh S.-H., Brook C., Governato F., Brinks E., Mayer L., de

Blok W. J. G., Brooks A., Walter F., 2011, AJ, 142, 24

Ostriker J. P., Steinhardt P., 2003, Science, 300, 1909

Peebles P. J. E., 2000, ApJ, 534, L127

Percival W. J., Reid B. A., Eisenstein D. J., Bahcall N. A., Budavari T., Frieman J. A., Fukugita M., Gunn J. E., Ivezić Ž. e. a., 2010, MNRAS, 401, 2148

Planck Collaboration Ade P. A. R., Aghanim N., ArmitageCaplan C., Arnaud M., Ashdown M., Atrio-Barandela F., Aumont J., Baccigalupi C., Banday A. J., et al. 2014, A\&A, 571, A16

Pontzen A. \& Governato F., 2014, Nature 506, 171.

Raveri M., 2015, ArXiv e-prints

Read J. I., Gilmore G., 2005, MNRAS, 356, 107

Reiprich T. H. \& Böhringer H., 2002, Astrophys. J. 567, 716.

Rodrigues D. C., Marra V., del Popolo A. \& Davari Z., 2018a, Nat. Astron. 2, 668.

Rodrigues D. C., Marra V., Del Popolo A. \& Davari Z., 2018b, Nat. Astron. 2, 927.

Romano-Díaz E., Shlosman I., Hoffman Y., Heller C., 2008, ApJ, 685, L105

Saburova A., Del Popolo A., 2014, MNRAS, 445, 3512

Sanders R. H. \& McGaugh S. S., 2002, Annu. Rev. Astron. Astrophys. 40, 263.

Skordis C. \& Zlosnik T., arXiv:1905.09465

Sommer-Larsen J., Dolgov A., 2001, ApJ, 551, 608

Spergel D. N., Verde L., Peiris H. V., Komatsu E., Nolta M. R., Bennett C. L., Halpern M., Hinshaw G., Jarosik N., Kogut A., Limon M., Meyer S. S., Page L., Tucker G. S., Weiland J. L., Wollack E., Wright E. L., 2003, ApJS, 148, 175

Starobinsky A. A., 1980, Physics Letters B, 91, 99

Stone C. \& Courteau S., arXiv:1908.06105

Tan A. et al. [PandaX-II Collaboration], 2016, Phys. Rev.
Lett. 117, 121303.

Trujillo I. et al., 2019, Mon. Not. R. Astron. Soc. 486, 1192. van Dokkum P. et al., 2018, Nature 555, 629.

van Dokkum P., Danieli S., Abraham R., Conroy C. \& Romanowsky A. J., 2019a, Astrophys. J. 874, L5.

van Dokkum P. et al., 2019b, Astrophys. J. 880, 91.

Verlinde E. P., 2017, SciPost Phys. 2, 016.

Vikhlinin A., Kravtsov A., Forman W., Jones C., Markevitch M., Murray S. S. \& Van Speybroeck L., 2006, Astrophys. J. 640, 691.

Weinberg S., 1989, Reviews of Modern Physics, 61, 1

Zentner A. R., Bullock J. S., 2003, ApJ, 598, 49

Zhao H.-S. \& Famaey B., 2012, Phys. Rev. D 86, 067301.

Zhou Y., Zhao Z.-C., Chang Z., 2017, Astrophys. J., 847, 86 